\title{
Broadband Noise Prediction When Turbulence Simulation Is Available- Derivation of Formulation 2B and its Statistical Analysis
}

\author{
F. Farassat and Jay Casper \\ NASA Langley Research Center \\ Hampton, Virginia
}

\begin{abstract}
We show that a simple modification of Formulation 1 of Farassat results in a new analytic expression that is highly suitable for broadband noise prediction when extensive turbulence simulation is available. This result satisfies all the stringent requirements, such as permitting the use of the exact geometry and kinematics of the moving body, that we have set as our goal in the derivation of useful acoustic formulas for the prediction of rotating blade and airframe noise. We also derive a simple analytic expression for the autocorrelation of the acoustic pressure that is valid in the near and far fields. Our analysis is based on the time integral of the acoustic pressure that can easily be obtained at any resolution for any observer time interval and digitally analyzed for broadband noise prediction. We have named this result as Formulation 2B of Farassat. One significant consequence of Formulation 2B is the derivation of the acoustic velocity potential for the thickness and loading terms of the Ffowcs Williams-Hawkings (FW-H) equation. This will greatly enhance the usefulness of the Fast Scattering Code (FSC) by providing a high fidelity boundary condition input for scattering predictions.
\end{abstract}

\section{1- Introduction}

Broadband noise prediction of rotating machinery and airframes is an important problem of aeroacoustics. The most successful methods today are semiempirical $[1,2]$ that depend on some measured data to find the constants required in the model. We believe that semiempirical methods will be useful for the foreseeable future because of their ease of usage and speed of execution on a computer. Broadband noise is generated by the random fluctuations of the surface pressure as well as velocity fluctuations on the data surface when the governing equa- 
tion of noise generation is $\mathrm{FW}-\mathrm{H}_{\mathrm{pds}}$. Here the subscript $p d s$ stands for penetrable (permeable or porous) data surface. Turbulence in the boundary layer as well as in the medium around the moving body, therefore, plays a significant role in generation of the broadband noise. So far, the detailed information on the surface pressure and velocity fluctuations has not been available. However, we feel that the trend today is toward heavy use of computers for turbulence simulation in the future [3-7]. We, therefore, are going to assume that we have extensive turbulence simulation at our disposal and ask ourselves what would be a suitable acoustic formulation to use for prediction of broadband noise of rotating machinery and airframes. We believe that we can answer this question fully in the present paper. Surprisingly, the answer lies in the use of a time domain method. There is also an unexpected byproduct of our analysis that has an impact in acoustic scattering computations.

Time domain methods have rarely been used in broadband noise prediction in the past. We are only aware of a few publications by Casper and Farassat [8- 9] on this subject. These authors used a time domain result called Formulation 1B of Farassat [8]. They then reproduced two sets of broadband noise predictions by Amiet in frequency domain for which experimental acoustic data were available $[10,11]$. Comparison of acoustic predictions in time domain with both of Amiet's predictions and measured data was excellent. Although these studies by Casper and Farassat verified the feasibility and suitability of time domain analysis in broadband noise prediction, Formulation 1B suffers from some restrictions that do not meet our stringent requirements for derivation of a useful acoustic formula for noise prediction. Below we present these requirements and the weaknesses of Formulation 1B. We will then demonstrate that it is possible to derive a new time domain result for broadband noise prediction that meets all of our requirements for a good predictive formula.

To improve broadband noise prediction, a physics based methodology based on FW-H or $\mathrm{FW}-\mathrm{H}_{\mathrm{pds}}$ equations is proposed here assuming that the detailed pressure and velocity fluctuations are known on or near the blade (FW-H eq. [12]) or on the data surface (FW $-\mathrm{H}_{\mathrm{pds}}$ eq. [13]). The Aeroacoustics Branch of NASA Langley Research Center has a long history of deriving formulations for rotating blade and airframe noise prediction in the time domain (Formulation 1, 1A, etc.). The first author as well as Kenneth S. Brentner (now a Professor of Engineering at Penn State University) have been involved in most of these developments. Throughout these years, NASA Langley researchers followed a technical philosophy that has proven to lead to highly useful acoustic formulations. A set of stringent requirements for acceptance of a good predictive acoustic formula underlie the Langley philosophy. These requirements are:

1- There must be no restrictions on the geometry of the noise generator (blades, airframe, etc.). This means that results for infinitely thin blades are not acceptable to us.

2- There must be no restrictions on the kinematics of the noise generator. This means that the blade cannot be restricted to a straight line motion or to lie on a helicoidal surface.

3- The result must be valid in the near and far fields.

4- One should be able to calculate the noise for an observer that is stationary in the medium or in motion with the aircraft.

We add one more requirement for an acoustic formula to be suitable for statistical analysis after all the above requirements are met. We would like the formula to be composed of one 
term. This can only be appreciated from the authors' work reported in [14]. From this work, one can see that developing statistical results based on Formulation 1A of Farassat [15, 16] when the near field terms are retained becomes very complicated even when the body moves rectilinearly. The problem can be traced to the fact that we have multiple terms in Formulation 1A. We also conclude that Formulation 1B does not meet our requirements because it is for an infinitely thin airfoil moving rectiliearly with the observer located in the far field. Nevertheless, the assumptions we used in deriving Formulation 1B corresponded exactly with the assumptions that Amiet used in his analysis in frequency domain $[10,11]$. The work by Casper and Farassat proved that time domain methods can be used for broadband noise prediction of rotating blades and airframes.

\section{2- Derivation of Formulation 2B}

Our governing equation is the $\mathrm{FW}-\mathrm{H}$ or $\mathrm{FW}-\mathrm{H}_{\mathrm{pds}}$ equation without the quadrupole term. Let the moving surface be defined by $f(\boldsymbol{x}, t)=0, f>0$ outside the body. Here the body can be a solid surface, e.g., a wing or a blade, or a penetrable (permeable, porous) data surface. Let $\rho^{\prime}$ be the density perturbation and define $p^{\prime}=c^{2} \rho^{\prime}$, with $c$ being the speed of sound in the undisturbed medium. The FW-H equation is [12]:

$\square^{2} c^{2} \rho^{\prime}=\square^{2} p^{\prime}=\frac{\partial}{\partial t}\left[\rho_{0} v_{n} \delta(f)\right]-\frac{\partial}{\partial x_{i}}\left[p n_{i} \delta(f)\right]$,

and the $\mathrm{FW}-\mathrm{H}_{\mathrm{pds}}$ is [13]

$\square^{2} p^{\prime}=\frac{\partial}{\partial t}\left[\rho_{0} U_{n}\right] \delta(f)-\frac{\partial}{\partial x_{i}}\left[L_{i} \delta(f)\right]$.

In Eq. (2), we have used the following two symbols defined by the relations

$U_{n}=\left(1-\frac{\rho}{\rho_{0}}\right) v_{n}+\frac{\rho u_{n}}{\rho_{0}}$

and

$L_{i}=p \delta_{i j} n_{j}+\rho u_{i}\left(u_{n}-v_{n}\right)$

In these equations, $\rho_{0}$ is the density of the undisturbed medium, $v_{n}$ and $p$ are the normal velocity and pressure on the body, respectively. We have used the symbols $\rho$ and $u_{n}$ as the fluid density and velocity with respect to the frame fixed to the undisturbed medium, respectively. The component of the unit outward normal to the moving body is $n_{i}, \delta(f)$ is the Dirac delta function and $\delta_{i j}$ is the Kronecker delta.

Without loss of generality, we work with the solution of FW-H equation retaining the thickness term involving $v_{n}$, the local normal velocity of the surface, which in the case of using $\mathrm{FW}-\mathrm{H}_{\mathrm{pds}}$ equation, in the form of the term $U_{n}$ of Eq. (3), contributes to the broadband noise because this term includes the random fluctuations of fluid particle velocity on the data surface. Formulation 1 of Farassat is a solution of Eq. (1) for a body in subsonic motion [15, 16]: 
$4 \pi p^{\prime}(\boldsymbol{x}, t)=\frac{\partial}{\partial t} \int_{f=0}\left[\frac{\rho_{0} v_{n}}{r\left(1-M_{r}\right)}+\frac{p \cos \theta}{c r\left(1-M_{r}\right)}\right]_{r e t} d S+\int_{f=0}\left[\frac{p \cos \theta}{r^{2}\left(1-M_{r}\right)}\right]_{r e t} d S$

Here the observer space-time coordinates are $(\boldsymbol{x}, t), p=p(\boldsymbol{y}, \tau)$ is the local surface pressure at the source space-time coordinates $(\boldsymbol{y}, \tau)$, and $r=|\boldsymbol{x}-\boldsymbol{y}|$ is the distance between the source and the observer at the emission time. The symbol $M_{r}$ stands for the Mach number of the source in the radiation direction at the emission time. The angle $\theta$ is between the direction $\boldsymbol{r}=\boldsymbol{x}-\boldsymbol{y}$ and local normal to the surface. Figure 1 shows the description of some of these variables. The subscript ret in Eq. (5) stands for the retarded or emission time which in general is obtained from the solution of the equation $c(\tau-t)+|\boldsymbol{x}-\boldsymbol{y}(\boldsymbol{\eta}, \tau)|=0,(\boldsymbol{x}, t)$ is kept fixed here and $\boldsymbol{\eta}$ is the Lagrangian variable of the source on the blade or airframe for which the emission time is computed. It is important to recognize that the observer and source variables, $\boldsymbol{x}$ and $\boldsymbol{y}$, are specified in a frame fixed to the undisturbed medium while the variable $\boldsymbol{\eta}$ is generally specified in a frame fixed to the blade or airframe.

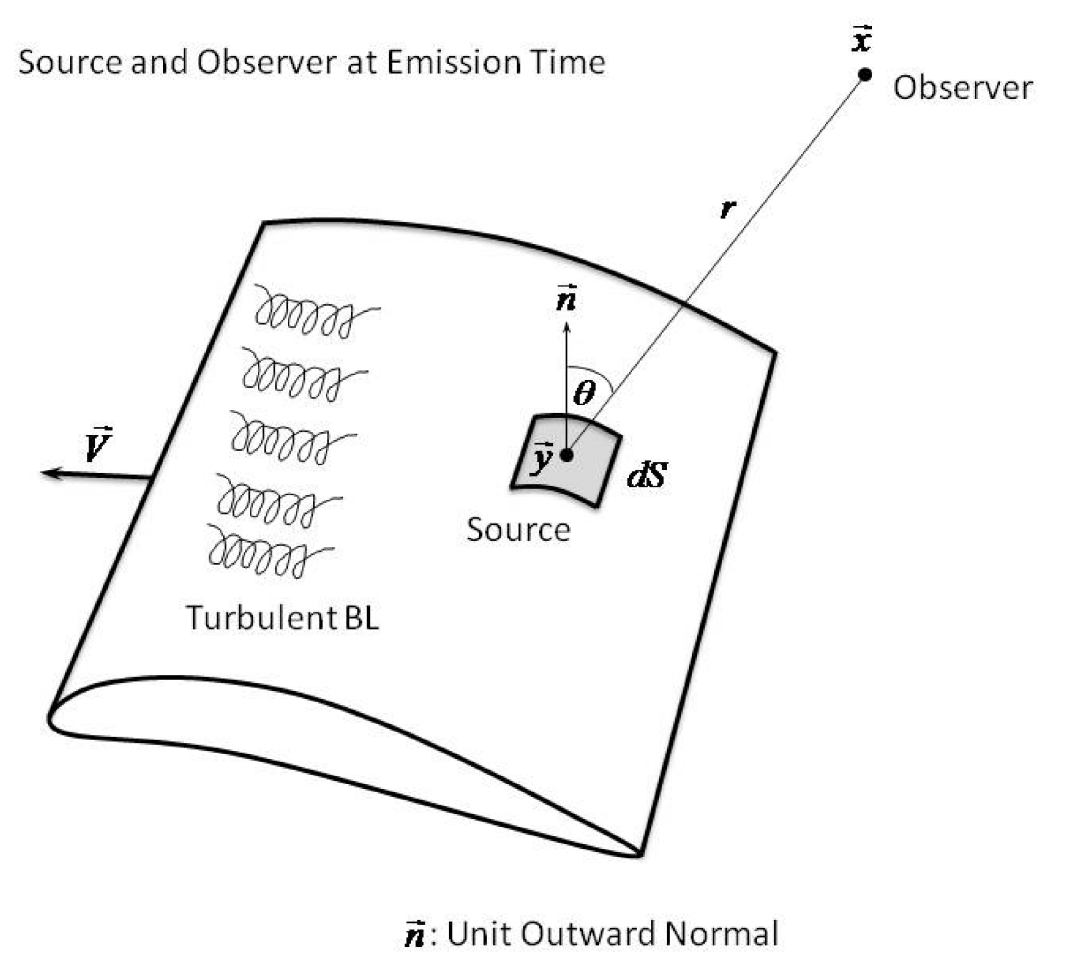

Figure 1- A moving blade or wing as seen by an observer fixed to the medium at the emission time of the source at $\boldsymbol{y}$. Note that the position of the source in a frame fixed to the moving body is described by $\boldsymbol{\eta}$.

When a moving body is in uniform rectilinear motion, as is often the case in airframe noise problems, the retarded time can be analytically calculated by a geometric construction known as the Garrick triangle shown in Figure 2 below. This can result in considerable savings of 
computation time.

Radiation process as seen from a frame fixed to the medium.

Triangle $A B C$ is known as the Garrick Triangle.

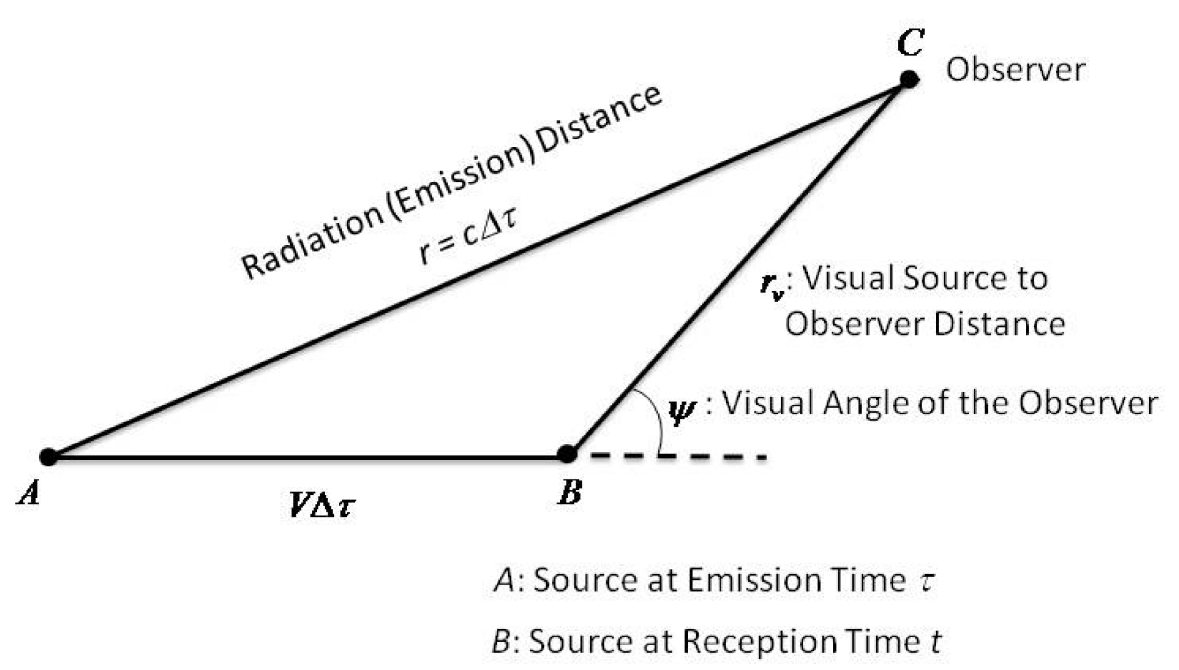

Figure 2- Computation of emission time and distance using the Garrick triangle. Note that we generally know the visual distance $r_{v}$ and the visual angle $\psi$. The radiation distance $r$ can be computed by application of cosine law to the triangle $A B C$ from which $\triangle \tau=r / c$ can be computed to get the emission time $t-\Delta \tau$.

We will now write Eq. (5) as follows:

$$
\begin{aligned}
& 4 \pi p^{\prime}(\boldsymbol{x}, t)= \\
& \frac{\partial}{\partial t}\left[\int_{f=0}\left[\frac{\rho_{0} v_{n}}{r\left(1-M_{r}\right)}+\frac{p \cos \theta}{c r\left(1-M_{r}\right)}\right]_{r e t} d S+\int_{0}^{t} d t^{\prime} \int_{f=0}\left[\frac{p \cos \theta}{r^{2}\left(1-M_{r}\right)}\right]_{r e t} d S\right]
\end{aligned}
$$

Here we assume that in the inner integral of the second term on the right, the observer time is denoted as $t^{\prime}$ and that the body started to move at the time $t^{\prime}=0$. We now write the acoustic pressure in the following form:

$p^{\prime}(\boldsymbol{x}, t)=\frac{\partial \Pi(\boldsymbol{x}, t)}{\partial t}$

where we have defined a new symbol as follows 


$$
\Pi(\boldsymbol{x}, t)=\frac{1}{4 \pi}\left[\int_{f=0}\left[\frac{\rho_{0} v_{n}}{r\left(1-M_{r}\right)}+\frac{p \cos \theta}{c r\left(1-M_{r}\right)}\right]_{r e t} d S+\int_{0}^{t} d t^{\prime} \int_{f=0}\left[\frac{p \cos \theta}{r^{2}\left(1-M_{r}\right)}\right]_{r e t} d S\right]
$$

We will call Eq. (7) Formulation 2B (B for broadband) of Farassat. We propose to use rectangular integration in time in the second integral of Eq. (8) because for broadband noise calculations, the observer time step $\Delta t^{\prime}$ is a small fraction of the period of the highest frequency of interest. The rectangular integration in time that we propose is consistent with the current practice of digital signal analysis [17]. We note that the function $\Pi(\boldsymbol{x}, t)$ can be calculated by any noise prediction code such as WOPWOP [15] or ASSPIN [18] just as the acoustic pressure is with little computational effort. It is important to note that we do not intend to use Eq. (7) for calculation of the acoustic pressure signature which in the case of broadband noise is devoid of useful information without further analysis. We propose that the quantity $\Pi(\boldsymbol{x}, t)$ be computed numerically for a given observer time interval at discrete intervals and advanced signal processing techniques be applied to these digital data to get the spectral properties of the broadband noise. Figure 3 shows the flow diagram of the proposed method for the loading term of the FW-H equation.

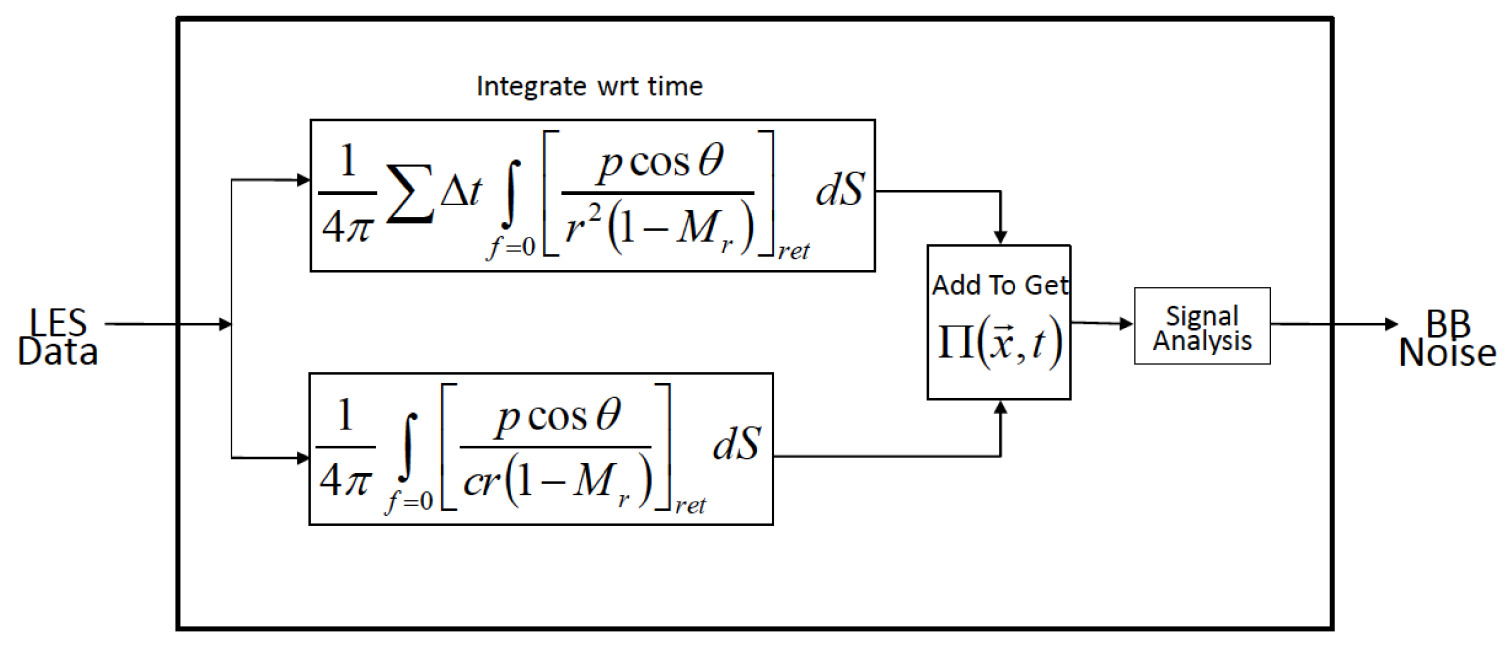

Figure 3- The flow diagram of the proposed method of using Formulation 2B shown only for the loading noise term of the FW-H equation

Finally, note that Formulation 2B satisfies all of the requirements of a good noise prediction formula that we mentioned in the Introduction. In particular, near field computation of broadband noise is possible from this formula as well as the inclusion of the motion of the blade or the airframe. The most striking property of Formulation $2 \mathrm{~B}$ is that the input data does not have to be differentiated in time (as in Formulation 1A) or in space (as in Formulation 1B and the supersonic Formulation 3 used in ASSPIN). This means that the turbulence simulation data can be used without further manipulation and, thus, the accuracy and speed of noise prediction are increased significantly. We do not recommend Eq. (7) to be used for discrete frequency noise prediction of rotating blades and airframes for which Formulation 1A is recommended 


\section{$[15,16]$.}

To find the spectrum of the acoustic pressure signature, we take the Fourier transform of Eq. (7) resulting in:

$\hat{p}^{\prime}(x, f)=2 \pi$ if $\hat{\Pi}(x, f)$

where $f$ now stands for the frequency of the sound and we have defined the Fourier transform of $\Pi(\boldsymbol{x}, t)$ as follows

$\hat{\Pi}(x, f)=\int_{-\infty}^{\infty} e^{-2 \pi i f t} \Pi(x, t) d t$

The fast Fourier transform (FFT) is the most appropriate tool to be used here on the digital data $\Pi(\boldsymbol{x}, t)$.

We note that the surface terms of $\mathrm{FW}-\mathrm{H}_{\mathrm{pds}}$ equation are similar to those of $\mathrm{FW}-\mathrm{H}$ equation. The fluctuation of fluid velocity by turbulent eddies are included in the $U_{n}$ term of the $\mathrm{FW}-\mathrm{H}_{\mathrm{pds}}$ equation. These velocity fluctuations can contribute significantly to the broadband noise generation.

\section{3- Statistical Analysis}

We find the autocorrelation of the acoustic pressure based on Eq. (7). We have, for a sufficiently large time period $T$

$$
\begin{gathered}
R_{p^{\prime}}(\boldsymbol{x}, \tau)=\frac{1}{T} \int_{0}^{T} p^{\prime}(\boldsymbol{x}, t) p^{\prime}(\boldsymbol{x}, t+\tau) d t=\frac{1}{T} \int_{0}^{T} \frac{\partial \Pi(\boldsymbol{x}, t)}{\partial t} \frac{\partial \Pi(\boldsymbol{x}, t+\tau)}{\partial t} d t= \\
\frac{1}{T} \int_{0}^{T} \frac{\partial \Pi(\boldsymbol{x}, t)}{\partial t} \frac{\partial \Pi(\boldsymbol{x}, t+\tau)}{\partial \tau} d t=\frac{1}{T} \frac{\partial}{\partial \tau} \int_{0}^{T} \frac{\partial \Pi(\boldsymbol{x}, t)}{\partial t} \Pi(\boldsymbol{x}, t+\tau) d t= \\
\frac{1}{T} \frac{\partial}{\partial \tau} \int_{0}^{T} \frac{\partial \Pi(\boldsymbol{x}, t-\tau)}{\partial t} \Pi(\boldsymbol{x}, t) d t=-\frac{1}{T} \frac{\partial}{\partial \tau} \int_{0}^{T} \frac{\partial \Pi(\boldsymbol{x}, t-\tau)}{\partial \tau} \Pi(\boldsymbol{x}, t) d t= \\
-\frac{1}{T} \frac{\partial^{2}}{\partial \tau^{2}} \int_{0}^{T} \Pi(\boldsymbol{x}, t-\tau) \Pi(\boldsymbol{x}, t) d t=-\frac{\partial^{2} R_{\Pi}(\boldsymbol{x}, \tau)}{\partial \tau^{2}}
\end{gathered}
$$

Here $R_{p^{\prime}}(\boldsymbol{x}, \tau)$ is the autocorrelation of the acoustic pressure and $R_{\Pi}(x, \tau)$ is the autocorrelation of the digital data $\Pi(x, t)$. We have assumed that we have a stationary random process here which is a reasonable assumption in broadband noise prediction. The variable $\tau$ in Eq. (11) is the time displacement of the autocorrelation function and is not related to the emission time. It is interesting to note that, in general, the autocorrelation function $R_{\Pi}(x, \tau)$ is a smooth function for broadband noise so that any good numerical differentiation method can be used to find the autocorrelation of the acoustic pressure.

It is obvious that more statistical analysis can be performed on Formulation 2B. For example, the spectral density of the broadband noise can be found by taking Fourier transform of Eq. (7) [17]. In all these analyses, one always works with the digital data $\Pi(x, t)$. Ideally, the 
prediction of the broadband noise as proposed here should correspond to the digital analysis of the measured acoustic data, thus, reducing the possibility of errors due to the differences in the interpretation of measured and predicted acoustic data.

\section{4- Further Analysis and Future Plans}

One surprising result following from Formulation $2 \mathrm{~B}$ is that we have now access to the acoustic velocity potential in the frame fixed to the medium in the near and far fields. Let $\phi(x, t)$ be the acoustic velocity potential. Then, from the relation

$$
p^{\prime}(\boldsymbol{x}, t)=\frac{\partial \Pi(\boldsymbol{x}, t)}{\partial t}=-\rho_{0} \frac{\partial \phi(\boldsymbol{x}, t)}{\partial t}
$$

we see that

$$
\phi(\boldsymbol{x}, t)=-\frac{1}{\rho_{0}} \Pi(\boldsymbol{x}, t)
$$

This is the acoustic velocity potential for the thickness and loading terms of the FW-H as well as $\mathrm{FW}-\mathrm{H}_{\mathrm{pds}}$ equations. To the authors' knowledge, it is the first time that this result has been derived. This is the subject of another paper by the first author, Mark H. Dunn and Ana F. Tinetti to be submitted for publication. The acoustic velocity is required to satisfy the boundary condition in the computation of the scattering of propulsion and airframe noise from aircraft wings and fuselage. The Fast Scattering Code (FSC) of NASA Langley developed by Dunn and Tinetti $[19,20]$ can now use this acoustic velocity potential in the near and far fields when the incidence noise is computed from any code based on FW-H or FW $-\mathrm{H}_{\mathrm{pds}}$.

The authors are planning to use the results of this paper to reproduce Amiet's acoustic predictions of broadband noise in the frequency domain $[10,11]$. Some excellent measured acoustic data by Amiet and coworkers are available in these publications. Following these calculations, the authors will study broadband noise from open rotors, helicopter rotors and airframes.

\section{5- Concluding Remarks}

In this paper we have derived a new formula for the prediction of broadband noise of rotors and airframes that satisfies all the stringent requirements we have set for a good noise prediction formula. We have named it Formulation 2B and it is strictly for broadband noise prediction when extensive turbulence simulation data are available. The formulation involves the calculation of a new quantity $\Pi(\boldsymbol{x}, t)$, the time integral of the acoustic pressure, that can be easily calculated by any of our noise prediction codes at NASA such as WOPWOP, ASSPIN and ANOPP [21]. This quantity can be calculated in any given observer time interval for small time increments. Advanced digital signal analysis will then be applied to $\Pi(\boldsymbol{x}, t)$ corresponding precisely to the analysis of the measured acoustic data to predict the broadband noise. An important fact to remember is that no time or space derivatives of the surface pressure or 
velocity fluctuations are required in Formulation $2 \mathrm{~B}$ and the turbulence simulation data are used as computed. As a matter of fact, an acoustic code can be developed that uses the grid structure of turbulence simulation code and thus avoiding the interpolation of turbulence data for the acoustic calculation.

\section{Acknowledgement}

The authors gratefully acknowledge the help of Drs. Mark Dunn and Douglas Nark in preparation of this paper.

\section{6- References}

1- T. F. Brooks and C. L. Burley: Rotor broadband noise prediction with comparison to model data, AIAA Paper 2001-2210 (May 2001)

2- T. F. Brooks, C. L. Burley, Blade wake interaction noise for a model main rotor, AHS Technical Specialists' Meeting for Rotorcraft Acoustics and Aerodynamics, Williamsburg, Virginia (Oct. 1997).

3- Veer N. Vatsa, David P. Lockard and Mehdi R. Khorrami, "Application of FUN3D Solver for Aeroacoustic Simulation of a Nose Landing Gear Configuration," 17th AIAA/CEAS Aeroacoustics Conference, 6 - 8 June 2011, Portland, Oregon (submitted for publication, 2011)

4- David Lockard, Mehdi Khorrami and Fei Li: High Resolution Calculation of a Simplified Landing Gear, AIAA-2004-2887, 10th AIAA/CEAS Aeroacoustics Conference, May 10-12, 2004, Manchester, UK

5- Michael L. Shur, Philippe R. Spalart and Michael Kh. Strelets: Noise prediction for increasingly complex jets. Part I: Methods and tests, International Journal of Aeroacoustics, 4 (3\&4), $2005,213-246$

6- Björn Greschner, Frank Thiele, Marc C. Jacob and Damiano Casalino: Prediction of sound generated by a rod-airfoil configuration using EASM DES and the generalised Lighthill/FW-H analogy, Computers \& Fluids 37, 2008, 402-413

7- Arne Stuermer and Jianping Yin: Low-Speed Aerodynamics and Aeroacoustics of CROR Propulsion Systems, AIAA 2009-3134, 15th AIAA/CEAS Aeroacoustics Conference, 11 - 13 May 2009, Miami, Florida

8- J. Casper and F. Farassat, A new time domain formulation for broadband noise predictions, International Journal of Aeroacoustics 1(3), 2002, 207-240

9- J. Casper and F. Farassat: Broadband trailing edge noise predictions in the time domain, Journal of Sound and Vibration, 271, 2004, 159-176

10- Paterson, R. W. and Amiet, R. K.: Noise and Surface Pressure Response of an Airfoil to Incident Turbulence, AIAA Journal ofAircraft, 14(8), 1977,1 729-736 
11- R.H. Schlinker, R.K. Amiet: Helicopter rotor trailing edge noise, NASA Contractor Report No. 3470, 1981

12- J. E. Ffowcs Williams and D. L. Hawkings: Sound generated by turbulence and surfaces in arbitrary motion, Philosophical Transactions of the Royal Society, A264, 1969, 321-342

13- Kenneth S. Brentner and F. Farassat: Analytical Comparison of the Acoustic Analogy and Kirchhoff Formulation for Moving Surfaces, AIAA Journal, 1998, 36(8), 1379-1386

14- F. Farassat and J. Casper: Some analytic results for the study of broadband noise radiation from wings, propellers and jets in uniform motion, International Journal of Aeroacoustics, 2 (3 \& 4), 2003, 333-348

15- Kenneth S. Brentner: Prediction of Helicopter Discrete Frequency Rotor Noise- A Computer Program Incorporating Realistic Blade Motions and Advanced Formulation, NASA TM 87721, October 1986,

16- F. Farassat: Derivation of Formulations 1 and 1A of Farassat, NASA TM-2007-214853, March 2007

17- D. E. Newland: An Introduction to Random Vibrations, Spectral \& Wavelet Analysis, Third edition, 1993, Longman Scientific and Technical

18- F. Farassat, S. L. Padula and M. H. Dunn: Advanced Turboprop Noise Prediction Based on Recent Theoretical Results, Journal of Sound and Vibration, 119(1), 1987, 53-79

19- M. H. Dunn and A. F. Tinetti, A. F: Aeroacoustic Scattering Via the Equivalent Source Method, AIAA 2004-2937, presented at AIAA/CEAS Aeroacoustics Meeting in Manchester, May 2004.

20- A. F. Tinetti and M. H. Dunn: Scattering of High Frequency Duct Noise by Full Scale Hybrid Wing Body Configurations, AIAA-2009-3400, presented at AIAA/CEAS Aeroacoustics Meeting in Miami, May 2009

21- Casey L. Burley, Thomas F. Brooks, William M. Humphreys, John W. Rawls: ANOPP landing-gear noise prediction with comparison to model-scale data, International Journal of Aeroacoustics, 8(5), 2009, 445-475 